\title{
Las convenciones interpretativas como control horizontal de la decisión judicial ${ }^{1}$
}

Interpretative conventions as a way of horizontal control of judicial decision

\author{
Federico José Arena ${ }^{2}$
}

\begin{abstract}
Resumen
En este trabajo propongo conectar la discusión filosófica sobre la práctica judicial de identificación del derecho con el problema del control de la actividad judicial. Por control de la actividad judicial me refiero a la verificación de la corrección jurídica de las decisiones de jueces y juezas en sus sentencias acerca de la existencia y el contenido del derecho. Procuraré mostrar que, además de formas de control vertical, existe la posibilidad de un tipo de control horizontal apoyado en la estructura convencional que adquiere la acción colectiva de jueces y juezas. El texto se concentrará en dos cuestiones centrales: en qué consiste esa estructura convencional y de qué modo permite controlar la actividad judicial.
\end{abstract}

Palabras clave: decisión judicial - control horizontal - convenciones interpretativas - criterios de corrección

\begin{abstract}
In this paper I intend to connect the philosophical discussion on the judicial practice of identifying the law with the problem of how to control judicial activity. By control of judicial activity I mean the verification of the legal correctness of the decisions on the existence and content of the law taken by judges in their sentences. I will try to show that, in addition to forms of vertical control, there is the possibility of a type of horizontal control based on the conventional structure that the collective action of judges acquires. The text will focus on two main issues: the elements of the conventional structure and how it may be able to control judicial activity.
\end{abstract}

Keywords: judicial decision - horizontal control interpretative conventions - correctness criteria

\section{Derecho/ artículo científico}

Citar: Arena, F. J. (2021). Las convenciones interpretativas como control horizontal de la decisión judicial. Omnia. Derecho y sociedad, 4 (4), pp. 11-25.

\footnotetext{
1 El presente artículo se enmarca en el proyecto «Teorías y técnicas de la interpretación jurídica. Un análisis teóricopráctico de los cánones de interpretación vigentes», dirigido por Paula Gaido y Federico Arena, radicado en el Centro de Investigaciones Jurídicas y Sociales (CIJS), Facultad de Derecho, Universidad Nacional de Córdoba, Res. CIJS 30/2018.

2 Investigador adjunto del Consejo Nacional de Investigaciones Científicas y Técnicas de Argentina (CONICET). Abogado por la Universidad Nacional de Córdoba y doctor en Filosofía del Derecho y Bioética Jurídica por la Universidad de Génova, Italia. Sus líneas de investigación son la teoría general del derecho, la teoría de la interpretación y la relevancia jurídica de los estereotipos sociales. ORCID: 0000-0001-8826-635 3.fjarena@gmail.com
} 


\section{INTRODUCCIÓN}

La práctica judicial de identificación del derecho, es decir, el conjunto de acciones a través de las cuales los jueces determinan la existencia y el contenido del derecho, se ha encontrado al centro del debate iusfilosófico de los últimos años. Los filósofos del derecho han intentado elaborar esquemas diferentes para ofrecer una reconstrucción de esa práctica, con el objetivo de ofrecer una explicación de algunos problemas filosóficos, tales como la existencia de desacuerdos entre jueces, la pretensión de autoridad del derecho o la capacidad práctica de las normas jurídicas, entre otros. En particular han recurrido a modelos de acción colectiva construidos en ámbito económico (la teoría de los juegos) y en ámbito filosófico (hechos institucionales). Específicamente, se trata de formas en que la acción de un conjunto de individuos puede entrelazarse para formar una convención de coordinación (teoría de los juegos) (Lewis, 1969; Postema, 1982) o una convención constitutiva (hechos institucionales) (Marmor, 2009; Searle, 1995).

En este trabajo quisiera conectar esas discusiones con el problema del control de la actividad de los jueces; en particular, sus decisiones sobre la identificación del derecho. Generalmente, se considera que esa actividad (i.e., las decisiones de los jueces sobre la identificación del derecho) resulta controlada por mecanismos institucionales específicamente previstos a tal efecto, en particular los distintos recursos procedimentales. Estos recursos permiten que un órgano judicial, superior al que tomó la decisión, controle la corrección de la decisión del tribunal inferior. Se trata, en consecuencia, de un tipo de control vertical. Los límites de este control se manifiestan cuando se trata de órganos judiciales supre- mos, es decir, órganos cuyas decisiones son finales, en el sentido de que no existe un órgano superior que pueda revisarlas. Sin embargo, creo posible postular la existencia de un tipo de control horizontal apoyado en la estructura que adquiere la acción colectiva de los jueces; control que se extiende incluso a los órganos supremos. Intentaré ofrecer argumentos a favor de la tesis según la cual, en algunos casos, la acción colectiva de los jueces adquiere la estructura de una convención y esta estructura funciona como un tipo de control horizontal de las acciones de cada uno de ellos. Por convención entiendo una regularidad de comportamiento en la que (parte de) las razones por las que cada uno de los involucrados se conforma a la regularidad es que los demás lo hacen. Mi intención aquí no es tanto defender la efectiva existencia de tales convenciones, sino mostrar de qué modo, si se las acepta, es posible que funcionen como control de la actividad de los jueces.

Dividiré el trabajo en dos. Primero, procuraré mostrar en qué sentido se puede concebir la actividad de los jueces como regulada por convenciones. En este punto me referiré también al desafío escéptico según el cual no existe entre los jueces una convención. Segundo, indicaré de qué modo esas convenciones pueden funcionar como control de la acción colectiva de los jueces. En este punto abordaré las objeciones a la posibilidad de que las convenciones ofrezcan razones para la acción.

\section{CONVENCIONES Y ESCEPTICISMO}

\section{La noción de convención}

Aquí asumiré una noción estricta de convención. Es decir, en sentido amplio, algo que es convencional se opone a algo natural, ya que depende de los hombres, de sus acciones 
y creencias, u otras actitudes (Celano, 2010 [1997], pp. 281-282; Vilajosana Rubio, 2010, pp. 139-140) ${ }^{3}$. En el sentido estricto que usaré aquí, convenciones son solo una subclase de los hechos que dependen de las acciones y actitudes humanas. Se trata de hechos en los que las acciones y actitudes humanas adquieren una configuración particular. A este conjunto de circunstancias que deben darse para que exista una convención las llamaré condiciones de convencionalidad (Celano, 2010; Lewis, 1969; Marmor, 2009). Aquí, por razones de brevedad, me referiré solo a las que definen las convenciones no basadas en el acuerdo ${ }^{4}$ y las presentaré de un modo que parecerá estipulativo; sin embargo, cada una de ellas está apoyada en un argumento filosófico. La primera condición para la existencia de una convención no basada en el acuerdo es que se verifique una cierta regularidad o convergencia de comportamiento. Una regularidad de comportamiento puede ser concebida como «realizar la acción A en la ocasión O» o, puesto de modo más general, «A en $O$ ». La regularidad exige que $O$ se presente con cierta frecuencia, o al menos que se haya presentado más de una vez y que sea posible que vuelva a presentarse (Gilbert, 2008, p. 8). La segunda condición, que permite pasar de una mera convergencia de conducta a una convención, es una actitud de los involucrados, denominada por la literatura «condición de dependencia». Así, cuando se trata de una convención, existe un sentido crucial en el cual decimos que nos conformamos a la regularidad, en parte debido a que otros lo hacen. En particular, la razón del seguimiento está constituida (al menos en parte) por el hecho de que existe una práctica común (Celano, 2010 [2003], p. 330). Por ejemplo, si se concede que es convencional la práctica de que, al cortarse una comunicación telefónica, debe volver a llamar la persona que llamó primero, entonces la razón en virtud de la cual los involucrados se comportan regularmente de ese modo es, en parte, que los demás también lo hacen. Finalmente, la tercera condición para la existencia de una convención sin acuerdo es la arbitrariedad. Si bien no ha sido fácil precisar en qué consiste, aquí entenderé que la regularidad de comportamiento es arbitraria cuando los sujetos involucrados habrían podido seguir una regularidad alternativa, satisfaciendo la misma función (e.g., podría ser que quien deba rellamar sea la persona que recibió la llamada inicial) $)^{5}$. La posibilidad de elegir configurar de modo distinto una determinada convención puede advertirse claramente en aquellos casos donde ambas posibilidades se dan en el mundo actual. Por ejemplo, es sencillo advertir que el sentido de circulación de los automóviles es arbitrario, dado que existen lugares donde se conduce del lado izquierdo y lugares donde se conduce del lado derecho. Ambas opciones permiten realizar, entre otras, la misma finali-

\footnotetext{
${ }^{3}$ Esta distinción ha de ser matizada pues las acciones y las creencias humanas son parte de lo natural y, en consecuencia, también lo son las convenciones (véase Kekes, 1989, pp. 26-27).

${ }^{4}$ La identificación precisa de estas condiciones se debe, sobre todo, a David Lewis (véase Lewis, 1969). Desde mi punto de vista, la noción de convención basada en el acuerdo es secundaria respecto de aquella no basada en el acuerdo, aunque no podré extenderme aquí sobre esta última afirmación.

${ }^{5}$ La arbitrariedad no ha de ser confundida con la indiferencia. Ni tampoco la arbitrariedad implica indiferencia. (Véase Lewis, 1969, pp. 76-80; Marmor, 2009, pp. 8-9; Vilajosana Rubio, 2010, p. 176; Arena, 2011). Por ejemplo, podría ser que, dado el costo de la llamada, no sea indiferente quién tenga que rellamar.
} 
dad de organizar de manera segura el tráfico de automóviles ${ }^{6}$. En definitiva, las condiciones de existencia de las convenciones no basadas en el acuerdo consisten en la convergencia de comportamiento, la condición de dependencia y la arbitrariedad ${ }^{7}$.

Estas tres condiciones pueden combinarse con otras características y dar lugar a diferentes tipos de convenciones. Uno de ellos son las convenciones constitutivas ${ }^{8}$. La noción de convención constitutiva ha sido defendida por Andrei Marmor en distintos trabajos, pero es en su libro Social Conventions donde confiere un aspecto de unidad a su propuesta (Marmor, 2009). Existen actividades en las que los seres humanos suelen involucrarse con regularidad, que parecen satisfacer la condición de arbitrariedad $y$, además, parece existir un sentido en el que parte de las razones para involucrarse en tales actividades es que otras personas también lo hacen.

Estas actividades se presentan como regidas por un conjunto de reglas que poseen una doble función: (i) constituyen la práctica y (ii) regulan la conducta dentro de la práctica9 En otras palabras, definen la práctica y cómo participar en ella (Searle, 1969, p. 33). Asimis- mo, esas reglas (iii) definen o constituyen, en parte, los valores asociados con la práctica en cuestión y definen el discurso evaluativo a ella aplicable (Marmor, 2009, pp. 37-39).

Las convenciones constitutivas pueden entonces ser definidas como una regularidad de comportamiento(s) que adquiere(n) su significado a partir de reglas que definen una práctica social y el modo de participar en ella.

Ahora bien, aun cuando aceptemos esta reconstrucción de la noción de convención, resulta a primera vista dificultoso extenderla a la actividad de los jueces. Ello es así en cuanto es común que los jueces identifiquen de maneras muy diferentes el contenido del derecho. Es sobre este hecho que se apoya lo que llamaré el desafío escéptico. Si el escéptico tiene razón entonces es falso que exista una convención, pues para esto último es necesario que se dé una regularidad de comportamiento o convergencia.

\section{El desafío escéptico}

La interpretación jurídica es la actividad que consiste en identificar el derecho, es decir, la actividad de determinar el significado de los enunciados de las fuentes, i.e. de los textos normativos (Guastini, 2012, p. 27; Guastini, 2014, p.

${ }^{6}$ Para ser una alternativa, la regularidad debe satisfacer ciertas condiciones, al respecto véase Lewis, 1969, pp. 71 y Marmor, 2009, p. 10.

${ }^{7}$ Existe una propiedad ulterior que ha sido propuesta como condición de convencionalidad, a saber, el conocimiento común. Desde mi punto de vista, el conocimiento común no es una condición de convencionalidad, pero no entraré en esa discusión aquí. En igual sentido, véase Burge, 1975, p. 250; contra Celano, 2010 [1995].

${ }^{8}$ El otro tipo son las convenciones de coordinación introducidas, si bien no con esta denominación, por David Lewis (véase Lewis, 1969).

${ }^{9}$ Las convenciones constitutivas son entonces actividades constituidas por un conjunto de reglas. Para caracterizar estas reglas, Marmor recurre a la distinción de Searle entre reglas regulativas y reglas constitutivas (véase Searle, 1964 y, con mayor elaboración, Searle, 1969). Según Searle, las reglas regulativas son aquellas que regulan formas de conducta que existen independientemente de, y con anterioridad a, la creación de la regla (p. ej. «obligatorio llevar corbata en los exámenes»). Las reglas constitutivas, en cambio, son aquellas que crean o definen nuevas formas de conducta («marcar un gol»). Nótese que la capacidad de constituir conductas no es una propiedad de reglas individuales y aisladas sino de conjuntos o sistemas de reglas. Un sistema de reglas es constitutivo si define las condiciones de posibilidad de una actividad o práctica social. 
31 y ss.). El resultado de esta actividad consiste en (la formulación de) un enunciado interpretativo de la forma «D significa N», donde D está por el texto normativo (la disposición) y $\mathrm{N}$ por su significado (la norma) (Guastini, 2012, p. 45). Según el escéptico, no es posible determinar la corrección de los enunciados interpretativos. En palabras de Guastini:

El escepticismo ante las reglas, tal como lo concibo, equivale a sostener que cualquier tesis sobre la existencia de una única interpretación correcta -cualquier tesis que sostenga que los problemas interpretativos admiten una única respuesta "correcta» (i.e. verdadera) - es falsa. Y es falsa precisamente debido a la indeterminación de sistema jurídico en el sentido especificado, lo que trae como consecuencia que los enunciados interpretativos no tienen valor de verdad. La indeterminación interpretativa del sistema jurídico es el verdadero fundamento del escepticismo ante las reglas (Guastini, 2012, p. 38).

El fundamento principal de la tesis escéptica, según Guastini, es que los significados, que pueden en abstracto ser atribuidos a una disposición, son por lo general numerosos (o, al menos, más de uno). Siempre según el escéptico, este rasgo del significado de los textos jurídicos depende menos del carácter «objetivamente» equívoco del lenguaje que de otras fuentes tales como la multiplicidad de métodos interpretativos, las distintas teorías dogmáticas y el sentimiento de justicia de los intérpretes, i.e., sus preferencias éticas y políticas (Guastini, 2012, p. 40) $^{10}$.
Para el escéptico, dado que cada texto normativo puede ser, y de hecho es, interpretado en numerosas maneras diversas, no tiene sentido decir que un intérprete se ha equivocado en asociar al texto una norma (falsa), en lugar de otra norma (verdadera). Esto implica que no existen criterios (objetivos) que permitan predicar error respecto de los enunciados interpretativos $y$, por lo tanto, que toda afirmación de este tipo no es más que una expresión de la ideología o actitudes valorativas del intérprete que no tiene nada que ver con una convención. La interpretación introduce así un margen de indeterminación respecto del contenido del derecho que impide determinar si los jueces han seguido o no una convención. Si no es posible determinar cuándo una convención ha sido seguida o no, entonces no tiene sentido (i.e., es imposible) hablar con sentido de una convención.

El siguiente ejemplo permite ilustrar lo que sostiene el escéptico. El inciso 6 del artículo 163 del Código Penal argentino agrava la pena del hurto cuando «fuere de vehículos dejados en la vía pública o en lugares de acceso público». Al menos dos veces la Cámara Nacional de Casación Penal tuvo que decidir en recursos interpuestos por condenados en primera instancia por hurto agravado en virtud de haberse apoderado de una bicicleta (satisfaciendo las demás condiciones típicas). En esos casos, el recurrente sostenía que el término «vehículo» incluido en ese inciso no comprende a las «bicicletas». El 16 de febrero del año 2001, la Sala III de la Cámara Nacional de Casación Penal sostuvo, en la causa Vilchez, que «la bicicleta, debe considerarse incluida en el concepto de vehículo del inc. 6 del art. 163 del C.P.. ${ }^{11}$. Unos

\footnotetext{
${ }^{10}$ Véase, con algunas variaciones, Guastini, 2014, p. 62.

${ }^{11}$ Cámara Nacional de Casación Penal, Sala III, Causa n. 2884 «Vilchez, Carlos Abelardo s/ recurso de casación», sentencia del 16 de febrero de 2001.
} 
años más tarde, el 20 de noviembre de 2012, la Sala Il de la misma Cámara sostuvo, en la causa Benítez, que respecto del alcance del vocablo vehículo utilizado en la ley es menester «concluir en la exclusión de este medio del ámbito de la agravación punitiva» ${ }^{12}$.

Se trata de dos decisiones válidas dentro del ordenamiento jurídico argentino $y$, sin embargo, cada una atribuye a una misma disposición normativa (término "vehículo» del inciso 6 del artículo 163 del Código Penal) dos significados completamente opuestos (comprende o no a las bicicletas).

Esto muestra, creo sostendría el escéptico, que los métodos interpretativos disponibles en la cultura jurídica argentina permiten a los jueces atribuir una misma disposición significados diferentes por lo que, en definitiva, cuál de esos significados sea asociado al texto normativo dependerá de una decisión de los jueces mismos. En efecto, por un lado, la Sala III en la causa Vilchez se apoyó, entre otros, en dos métodos o técnicas interpretativas centrales. Primero, en el método literal formal, es decir, basado en lo señalado por los diccionarios de la lengua:

... la palabra vehículo en su definición académica no hace referencia a bicicleta; ni en la explicación de bicicleta se menciona «vehículo» pero sí velocípedo, y en este vocablo el Diccionario de la Real Academia Española dice que es un vehículo (21a. edición, Madrid, 1992); en consecuencia, semánticamente la bicicleta es un vehículo y en este vocablo están comprendidas las bicicletas (Cámara Nacional de Casación Penal, Sala III, 2001).
Segundo, en el método intencionalista, apoyado en las intenciones aplicativas (i.e., acerca de alcance del término) del legislador histórico. Así, citando las actas del debate parlamentario se indica que el legislador Villarroel sostuvo que

... con este proyecto se amplía la protección penal, no circunscribiéndola exclusivamente a los vehículos de cuatro ruedas o más. Vale decir a toda clase de vehículos. [...] desde el punto de vista de la política criminal que tanto vale o puede valer la propiedad de un automóvil como la de una bicicleta (Cámara Nacional de Casación Penal, Sala III, 2001).

Por otro lado, la Sala II, en Benítez, se apoyó a su vez en dos técnicas o métodos interpretativos centrales, entre otros. Primero, también en el método literal; pero en este caso en un argumento literal más sofisticado apoyado en el uso (y conectado con un argumento valorativo acerca de la incidencia de la indeterminación). Sobre la base de este método se afirma que «bicicleta» no es un caso paradigmático de «vehículo», i.e. una bicicleta no es el primer ejemplo que se le viene a la mente a un usuario competente del lenguaje cuando piensa en el significado de la palabra «vehículo». Así como «usar como medio de pago» no es el significado que se le viene en mente a un usuario competente del lenguaje cuando piensa en el significado de «usar arma de fuego». Sobre esta base, y a partir de la consideración de que el derecho penal debe ser de aplicación restrictiva, se sostiene que no debería aplicarse a aquellos casos que no constituyen el significado paradigmático de los términos usados en el texto legal. El segundo método usado

\footnotetext{
${ }^{12}$ Cámara Nacional de Casación Penal, Sala II, Causa n. 15.268 «Benítez Alvarez, Carlos Esteban s/recurso de casación», sentencia del 20 de noviembre del año 2012.
} 
por la Sala II en Benítez es también el del legislador histórico, pero en este caso basado en la intención como finalidad (i.e., aquello que el legislador pretendía lograr con la modificación legislativa) y se señala que «en efecto, de la lectura del debate parlamentario se puede colegir - sin mayor esfuerzo- que evidentemente la expresión 'vehículo' se refiere, sin lugar a dudas, a los automóviles».

En definitiva, cada sala, recurriendo a métodos interpretativos disponibles en la cultura jurídica argentina, interpreta de manera diferente una misma disposición. Esto no es más que un ejemplo, sostiene el escéptico, de la indeterminación del ordenamiento jurídico; en el sentido de que no sabemos qué normas pertenecen al ordenamiento («obligatorio castigar de manera agravada el hurto de bicicletas» 0 «prohibido castigar de manera agravada el hurto de bicicletas») hasta que un juez haya interpretado la disposición normativa y resuelto así la equivocidad producida por la variedad de métodos interpretativos disponibles. De ello se sigue que no es posible sostener que existe entre los jueces una convención de interpretar «vehículo» de un modo o el otro, o, puesto en otros términos, de interpretar la disposición del Código Penal de un modo u otro. No hay convención porque no hay regularidad, ya que las interpretaciones son dispares. $Y$, dado que no hay convención, tampoco hay criterios de corrección.

Desde mi punto de vista es falso que la divergencia en la identificación de las normas elimine toda posibilidad de que exista una convención. Por el contrario, de un análisis detallado de la actividad interpretativa surge que en ella existen un conjunto de convenciones que permiten, hasta cierto punto, determinar cuándo el texto mediante el cual se formula la norma general ha sido correctamente interpretado. El desafío escéptico pierde de vista una distinción entre dos tipos de convenciones. Por un lado, convenciones que fijan cuál es el resultado de la interpretación y, por otro lado, convenciones que determinan cómo llevar a cabo la actividad interpretativa. Aun cuando concedamos al escéptico que entre los jueces no existen convenciones del primer tipo, de ello no se sigue que tampoco existan convenciones del segundo.

\section{Convenciones interpretativas}

Existe un sentido evidente según el cual la interpretación depende de convenciones. Las palabras o los enunciados no poseen un significado natural o dado, por el contrario, son los individuos o usuarios quienes atribuyen significado a las marcas y a los sonidos. Si esto fuera todo lo que sostiene al respecto la tesis convencionalista, entonces sería una tesis pacífica pero, creo, poco interesante. Además, como muestra el ejemplo de la bicicleta, la posible determinación del significado sobre la base de las convenciones del lenguaje ordinario no se traslada necesariamente al contexto de la interpretación jurídica ${ }^{13}$. Pero, sobre todo, este convencionalismo evidente no daría cuenta de la complejidad de la práctica interpretativa en el ámbito jurídico. Como acabamos de ver, en ese ámbito se usan convenciones interpretativas que no se limitan a las del lenguaje natural, sino que por lo general son más complejas y variadas (Bayón Mohino, 2002, pp. 63-64). El significado literal u ordinario de un enunciado jurídico no agota el conjunto de convenciones que determinan el contenido del derecho. Además de las con-

\footnotetext{
${ }^{13}$ Acerca de la diferencia entre interpretación en contextos de conversación ordinaria e interpretación jurídica véase Guastini, 2012, pp. 46-50 y 196-197; y Celano, 2017.
} 
venciones semánticas del lenguaje ordinario, existe un conjunto de convenciones interpretativas jurídicas que fijan el procedimiento a través del cual los intérpretes pueden atribuir significado a los textos jurídicos.

En este sentido Chiassoni afirma que un código interpretativo (o código hermenéutico) consiste en un conjunto de directivas hermenéuticas. Estas directivas están estructuradas, a su vez, en diferentes niveles, por lo que encontramos directivas primarias ${ }^{14}$, directivas secundarias $^{15}$ y directivas axiomáticas ${ }^{16}$.

Cada código incluye así un conjunto de reglas que definen un cierto tipo de método interpretativo y establecen cómo usarlo. Estas reglas permiten evaluar, al menos de manera relativa, los resultados interpretativos. Es por ello que puede afirmarse que tales códigos son convenciones constitutivas. Esto permite también dar cuenta del hecho de que los jueces, al interpretar un cierto texto en un determinado sentido, no lo hacen por lo general de manera aislada y sobre la base de métodos interpretativos de creación individual. Por el contrario, el hecho de que un determinado método sea utilizado por los demás jueces es una razón para usarlo. Ciertamente, cuál sea, de hecho, el contenido de cada código hermenéutico usado en una comunidad jurídica y qué tipos y niveles de directivas incluya, es una cuestión de hecho.
Aun cuando el escéptico tiene razón al afirmar que la pluralidad de los métodos interpretativos desempeña un papel relevante en la indeterminación del ordenamiento, de ello no se sigue que no existan criterios de corrección de la interpretación. Los métodos interpretativos forman parte de las convenciones sobre la base de las cuales los jueces asocian un significado a una disposición. Estas convenciones establecen cuáles son, y cómo usar, los procedimientos para obtener ciertos resultados interpretativos. Por ejemplo, en el caso del método intencionalista usado por la Sala Il (y más allá del resultado de la interpretación siguiendo la versión sofisticada del método literal), puede señalarse que, si bien se pretende apoyar el razonamiento en la intención del legislador histórico, en ningún momento se citan fragmentos de las actas (como sí lo hace la Sala III), sino que se reconstruye lo que, desde el punto de vista del juez, sería la finalidad de un legislador ideal que satisfaga ciertas condiciones de racionalidad respecto de la necesidad de la reforma legislativa. Es decir, dado que la inclusión del inciso 6. en el artículo 163 del Código penal se debió a la necesidad de modificar el anterior régimen de agravamiento del robo de automotor, ya que establecía una pena excesiva, el juez concluye que el nuevo texto no puede sino referirse también a automotores. Pero no cita ningún fragmento de las

\footnotetext{
${ }^{14}$ Las directivas primarias «son aquellas directivas que indican a los intérpretes recursos sobre la base de los cuales una disposición puede ser traducida en una o más normas explícitas» (Chiassoni, 2011, p. 90). Un ejemplo de directivas de este tipo es el conjunto de directivas que conforma la interpretación lingüística, dentro del cual podemos encontrar la que impone atribuir a una disposición el significado que resulta del uso común de las palabras y de las reglas gramaticales de la lengua natural en la que está formulada.

${ }^{15}$ Las directivas secundarias «regulan el uso de las directivas primarias y establecen los criterios para evaluar la corrección [...] de las interpretaciones [...] obtenidas sobre la base de ésas» (Chiassoni, 2011, p. 111).

${ }^{16}$ Por último, las directivas axiomáticas constituyen «el conjunto (la base) de principios últimos — de axiomas— de un código hermenéutico, y consisten habitualmente en reglas finales, que prescriben qué objetivo (normalmente general) de política del derecho debe ser perseguido al interpretar las disposiciones» (Chiassoni, 2011, p. 134). Véase también Chiassoni, 2004, p. 63.
} 
actas que así lo señalen. Hay un sentido, entonces, en que se usó de manera equivocada el método del legislador histórico; ya que no se siguió la directiva de citar fragmentos de las actas del debate parlamentario.

De todos modos, los criterios de corrección establecidos por las convenciones interpretativas tienen límites, ya que no suele existir una convención última que desempeñe el papel de criterio último para elegir entre los diferentes métodos interpretativos. Esto es advertido por Nino, cuando señala que en "Gedes Hnos.», «Mórtola y Cía.», «Piccardo» y «Bergamino» la Corte sostuvo que la interpretación de la Constitución debe atenerse a su letra y a su tenor literal; sin embargo en "Bressani» se sostuvo que el valor mayor de la Constitución no está en su texto escrito sino en su obra práctica, realista, de conciliación de intereses que exige, para que se la siga cumpliendo, una interpretación flexible y elástica a fin de adaptarla a nuevas ideas y circunstancias. En este sentido, no debe perderse de vista la terminología usada (Nino, 1992, p. 105).

\section{CONVENCIONESY CONTROL SOCIAL}

Controlar implica guiar el comportamiento. Pero esto último ha sido, a su vez, entendido en al menos dos modos distintos. Por un lado, como la capacidad de motivar la acción. Es decir, como la capacidad de causar o dar lugar a la realización de una acción. Por otro lado, como la capacidad de justificar la acción o, más específicamente, a la capacidad de ofrecer razones para la acción justificadas. Dicho con otras palabras, para guiar el comportamiento de otros es posible, o bien ofrecer una razón que motiva la acción, o bien ofrecer una razón que justifica la acción. Ahora bien, es todavía posible distinguir entre dos tipos de razones justificativas: meras razones justi- ficativas y razones que crean una obligación o un deber. La distinción también se formula distinguiendo entre mere having a reason y having an obligation or a duty (Postema, 1982; Coleman, 2001, p. 367). Quizás un modo de dar cuenta de esta distinción es señalando que en el primer caso se trata de racionalidad instrumental $y$, en el segundo, de racionalidad fundamental o no instrumental. La primera hace depender la racionalidad de una acción en función de los deseos del agente. La segunda hace depender la racionalidad de una acción en función de valores objetivos y universales (Schiavello, 2010, pp. 30-36).

Hay un sentido en que las convenciones funcionan de este modo. Empecemos entonces por el primer significado de guiar el comportamiento ofreciendo un motivo para la acción. Como señalé al inicio, los involucrados en una convención se conforman a la regularidad porque los demás lo hacen. Pero ello explica solo en parte la acción. La explicación completa hará referencia al deseo en virtud del cual están interesados en ajustarse a la regularidad. Por ejemplo, la razón por la que, cuando se interrumpe una comunicación telefónica, las personas al teléfono siguen lar regularidad «rellama quien llamó primero» es que ambas desean retomar la comunicación y saben que de ese modo podrán hacerlo. Del mismo modo, las acciones de cada uno de los jueces involucrados en la interpretación de los textos normativos se explican, en parte, por el hecho que los demás jueces se ajustan a la regularidad. Los jueces están motivados por el interés de interpretar los textos jurídicos y la preferencia condicionada de usar un determinado método solo si los demás jueces también lo hacen. La acción de cada uno de los jueces que utilizan las directivas que definen y establecen cómo usar cada método interpretativo se explica, en parte, por el hecho que 
los demás jueces usan ese método interpretativo. Pero, de nuevo, ello explica solo en parte la acción. Como en toda convención constitutiva, una vez que el agente ha decidido participar en la práctica definida por la convención, el hecho de que ciertas reglas formen parte de ella constituye un motivo para que los agentes sigan esas reglas. De lo contrario, su deseo de participar en la práctica no resultaría satisfecho. Así, quien desea interpretar una disposición sobre la base de los métodos usados en una cierta comunidad jurídica tiene un motivo para aplicar las reglas que definen cada uno de los métodos en cuestión. Sin embargo, la explicación completa ha de recurrir a la decisión o el interés de cada individuo en participar en la práctica. La acción de quienes juegan según las reglas del ajedrez se explica en parte porque los demás jugadores juegan según esas reglas, pero la explicación completa exige hacer referencia al interés por embarcarse en un partido de ajedrez, e.g., ganar un premio, satisfacer la ambición del maestro, etc. Así, quien desea, en virtud de cierto interés, interpretar una disposición sobre la base de los métodos usados en una cierta comunidad jurídica, tiene un motivo para aplicar las reglas que definen cada uno de los métodos en cuestión, pero el uso de estos dependerá también de sus propios intereses.

Pero, ¿pueden las convenciones ofrecer razones justificativas para la acción? Varios autores han negado esta posibilidad. Así, según Dickson, del hecho de que aceptemos que sería absurdo que, para interpretar, un juez siguiese sus convicciones políticas o morales, incluso cuando el método interpretativo usa- do no fuese seguido por ningún otro juez, no se sigue que la práctica compartida proporcione al juez una razón para ajustarse a determinadas convenciones interpretativas.

The fact that other judges adopt a given set of [interpretative methods] in common is thus relevant to [...] judge's deliberations about what she should do in order to identify the law in her jurisdiction, but is relevant in allowing her to identify what is the [interpretative methods] in that legal system, rather than in giving her reasons why she should accept and follow that rule so identified (Dickson, 2007, p. 396).

Sin embargo, tal como entiendo a Dickson, me parece que su argumento principal es que la existencia de una convención no constituye el tipo correcto de razón justificativa. Ello en cuanto la existencia de convergencia de comportamiento no es una razón completa para la acción ${ }^{17}$. Es decir, la existencia de una convención justifica la acción solo si existe, además, otra razón subyacente para ajustar el comportamiento a la convención. Por ejemplo, que los demás conduzcan por la derecha es una razón para conducir por ese mismo lado, en virtud de que existen razones a favor de evitar daños a uno mismo y a los demás. La existencia de una convención genera razones para la acción, pero la existencia de estas razones «convencionales» depende de que existan, además, razones para la acción subyacentes que no son creadas por la convención misma. Ahora bien, esta respuesta nos pone frente al desafío de mostrar de qué modo, combinada

\footnotetext{
17 «Una razón operativa es aquélla que justifica una actitud práctica hacia una acción, la formación de una intención; una razón auxiliar es un juicio descriptivo que identifica el tipo de acción que tenemos una razón operativa para ejecutar; una razón completa es el conjunto de premisas no redundantes de un razonamiento práctico, lo que incluye siempre una razón operativa y una o varias razones auxiliares» (Bayón Mohino, 1991, p. 86).
} 
con otras razones, una convención puede imponer un deber y no solo motivar la acción ${ }^{18}$.

Existen dos propuestas para defender la idea de que las convenciones imponen un deber. Primero, las convenciones pueden generar deberes convencionales en virtud de su carácter constitutivo. Segundo, en algunos casos existen deberes políticos que, asociados a la existencia de una convención, conllevan a que estas últimas impongan deberes. Veamos ambas estrategias.

Las convenciones pueden dar lugar a deberes condicionales en virtud del carácter constitutivo de algunas de ellas ${ }^{19}$. Una convención constitutiva define una práctica y establece cómo participar en ella. Así, una vez que estamos embarcados en jugar una partida de ajedrez, la capacidad práctica de las reglas del ajedrez queda explicada por el hecho de que seguirlas es el único modo de jugar al ajedrez. Además, esta explicación tiene la ventaja de no recurrir a ninguna norma ajena a la convención ${ }^{20}$. Del mismo modo, una vez que los jueces aceptan utilizar ciertos métodos interpretativos es posible seguir tales convenciones interpretativas (constitutivas) para identificar las normas que imponen deberes $^{21}$. Un enunciado de este tipo depende de la existencia de convenciones constitutivas, aceptadas por los jueces, que impongan asociar a una disposición la norma que impone ese deber. Como adelanté, la capacidad práctica de las convenciones constitutivas es siempre condicional ${ }^{22}$. Un individuo está justificado a seguir las reglas de la práctica si existe una razón subyacente para participar en ella. Que esa justificación imponga un deber dependerá de consideraciones morales o políticas que no pueden nunca ser respondidas por las reglas del juego mismas. El seguimiento de los métodos interpretativos vigentes en una comunidad será obligatorio para los jueces solo si existe una razón justificativa en sentido fuerte que así lo imponga, pero ello no es establecido por la existencia de la convención misma (Marmor, 2001, p. 85). Ahora bien, en el caso de las convenciones constitutivas se verifica una situación particular, ya que estas convenciones constituyen no solo la práctica sino también parte de su valor. De este modo, las razones primarias para participar en la práctica pueden verse influenciadas

\footnotetext{
${ }^{18}$ Algunos autores sostienen que, aun cuando exista una convención, ello no podría dar cuenta de la formulación de enunciados de deber. Por ejemplo, según Coleman ello se debe a que los deberes son un tipo de razón que no puede ser extinguida por el destinatario o los destinatarios, mientras que las reglas convencionales, tal como las he definido, sí pueden serlo (Coleman, 2001, p. 370).

${ }^{19}$ Los autores a los que me referiré a continuación se preocupan, en realidad, por la naturaleza constitutiva de la regla de reconocimiento. Como en el resto de este trabajo, adapto sus argumentos al análisis de la tesis convencionalista aquí defendida.

${ }^{20}$ En este sentido, según Redondo, «we can explain genuine legal normativity as an irreducible social phenomenon which does not necessarily depend on critical morality» (Redondo, 2001, p. 167). Cabe aclarar que Redondo está aquí intentando construir una teoría de la normatividad del derecho basada no solo en la noción de regla constitutiva, sino en la más abarcadora teoría de la realidad social elaborada por Searle (véase Searle, 1995; y Searle, 2010)

${ }^{21}$ «Only when some basic constitutive rules are accepted, we can see legal dispositions as specific institutional facts» (Redondo, 2001, p. 172).

${ }^{22}$ "The 'ought' such rules prescribe engenders reasons for action only on the condition that one is initially committed to participating in the practice constituted by these same conventions» (Marmor, 2001, p. 30).
} 
por la práctica misma ${ }^{23}$. Es decir, el compromiso puede derivar, según Marmor (2001), de los valores que la práctica misma contribuye a constituir. Pero la capacidad práctica derivará siempre de esos valores, no de la convención misma ${ }^{24}$. Por ejemplo, el uso regular de ciertos métodos interpretativos otorga cierta previsibilidad a la práctica lo cual, en el contexto jurídico, es un valor.

La segunda estrategia sostiene que el deber de los jueces de seguir la convención deriva de ciertos general political-moral concerns (Postema, 1982; y Postema, 1987). Según este autor, es posible mostrar que las convenciones sobre cuya base los jueces identifican y aplican el derecho poseen capacidad práctica. Según este argumento, una vez que la convención se estabiliza, se genera en cada uno de los involucrados la expectativa de que los demás continuarán conformándose a ella. La creación de estas expectativas puede dar lugar a la obligación de seguir la convención, si se verifican ciertas condiciones. Las expectativas generadas por la acción de un individuo, aun cuando sean razonables, no imponen necesariamente a quien las generó la obligación de satisfacer$\operatorname{las}^{25}$. La obligación surge solo cuando se dan las dos circunstancias siguientes:

(i) when detrimental reliance on those expectations is, in a suitably broad sense, «induced» and

(ii) when the context in which expectations arise and are sustained is closely analogous to a cooperative enterprise for mutual benefit in which considerations of fair play require conformity (Postema, 1982, p. 180).

Estas dos condiciones permiten construir el argumento denominado del fair-play. Este argumento se aplica a actividades que se distinguen por ciertas características, entre las que podemos encontrar la existencia de beneficios mutuos y la aceptación voluntaria de tales beneficios. El argumento puede resumirse del modo siguiente:

(i) No todos los casos de frustración de expectativas son casos disvaliosos (injustos).

(ii) Existe un caso en el cual la frustración de expectativas es disvaliosa (injusta).

(iii) La frustración de expectativas es disvaliosa cuando la expectativa es frustrada por quienes la alentaron.

Desde este punto de vista, las convenciones pueden crear obligaciones, ya que la conformidad general genera expectativas acerca del hecho de que la conducta de cada uno de los involucrados se mantendrá en el tiempo. Existen, según Postema, dos circunstancias que hacen que sea obligatorio para los jueces cumplir con las convenciones sobre cuya base identifican el derecho (i.e. identifican los textos normativos y los interpretan). En primer lugar, se trata, en el caso del juez, de preferencias de origen profesional. En el sentido de que el desempeño de su profesión, i.e. la efectiva aplicación y ejecu-

\footnotetext{
${ }^{23}$ «[T] he reasons one may have for participating, that is, the reasons for playing the game, as it were, would normally rely on those values which are constituted by the conventions constituting the practice» (Marmor, 2001, p. 27).

${ }^{24}$ «Whether judges, other practitioners, or laymen, have any moral or other reasons to play the game or not, is a totally separate question [...] those reason cannot be prescribed by the social conventions themselves» (Marmor, 2001, p. 33)

${ }^{25}$ Como, en el caso mencionado por Postema, el hecho de que Kant al dar su paseo diario pasara por la ventana de una señora siempre a las cinco de la tarde y de que sobre esa base la señora haya generado una expectativa usando la aparición de Kant como modo de medir el tiempo, no produce para Kant la obligación de mantenerla. Al respecto, véase Postema, 1982, p. 180.
} 
ción del derecho, exige que los jueces coordinen sus esfuerzos. En particular, exige por un lado que mantengan la unidad y la integridad de los textos y de la interpretación, y por otro lado demanda que medien entre ellos y los ciudadanos. En segundo lugar, la relación entre jueces y ciudadanos, si bien interdependiente, es asimétrica. La actividad del juez tiene carácter autoritativo $y$, además, se trata de ejercer poder sobre otros ${ }^{26}$. Los jueces conciben, y reclaman a los demás que conciban, su actividad como autoritativa, lo que equivale a decir que inducen expectativas en los ciudadanos. Este tipo de actividad exige, según la doctrina de la responsabilidad política, que los jueces actúen sobre la base de una teoría pública general, que los constriña a la consistencia y que no permita esconder tras ella el propio interés. Según Postema, esta general public theory exige que el juez (i) tienda a la coherencia respecto de la actividad de los demás jueces y (ii) respete las expectativas razonables y legítimas de los ciudadanos con relación a su actividad.

De este modo, si es correcto el argumento según el cual las expectativas inducidas han de ser respetadas, lo ciudadanos están legitimados a esperar que los jueces se ajusten a las convenciones. Y recíprocamente, los jueces, bajo la teoría de la responsabilidad política, están obligados a respetar esas expectativas. Y, por lo tanto, tienen la obligación de respetar las convenciones sobre cuya base identifican el derecho. Claramente, para que este argumento funcione, el contenido de las «preocupaciones político-morales generales» no puede coincidir con el conjunto de creencias que una sociedad posee al respecto. Pues en este caso no se habría avanzado en la justificación de las convenciones sobre cuya base se interpreta el derecho, ya que ese conjunto de creencias no coincide necesariamente con las exigencias de la moral sustantiva. El punto importante es, de todos modos, que bajo esta concepción los jueces tienen el deber de ajustarse a las convenciones interpretativas solo en aquellos ordenamientos jurídicos que expresan preocupaciones político-morales correctas. Las convenciones no ofrecerían razones justificadas en aquellas prácticas interpretativas que no incluyen ese tipo de preocupaciones o que, incluso, incluyen objetivos político-morales equivocados o aberrantes ${ }^{27}$.

\section{CONCLUSIONES}

En este texto he intentado mostrar que las convenciones interpretativas permiten controlar la acción judicial en dos sentidos. En primer lugar, porque ofrecen criterios de corrección para evaluar el modo en que ha sido atribuido significado a una disposición normativa. En segundo lugar, porque bajo ciertas condiciones ofrecen a jueces y juezas razones para la acción.

De ello no se sigue que el escéptico esté completamente equivocado. $\mathrm{O}$, al menos, lo dicho aquí no tiene la intención de mostrar eso. Es cierto que las disposiciones pueden ser interpretadas de más de una manera y ello se debe, sobre todo, a la existencia dentro de la cultura jurídica de una multiplicidad de métodos interpretativos y a la inexistencia de criterios últimos de ordenación. Todo lo cual, desde

\footnotetext{
${ }^{26}$ En este punto Postema se aparta de las tesis más específicas de Fuller, a quién sigue respecto de la insistencia en la necesidad de dar cuenta no solo de la relación de los jueces entre sí, sino también de estos con los ciudadanos. Fuller sostiene que la relación es simétrica y que tanto jueces como ciudadanos se encuentran ligados por obligaciones de reciprocidad, que fijan su contribución a la empresa cooperativa del derecho (véase Fuller, 1969 [1964], pp. 204-209).
}

${ }^{27}$ Esta es la crítica que, con algunas variaciones, Shiner dirige a Postema y Finnis (Shiner, 2010, p. 439). 
mi punto de vista, exige conceder al escéptico la tesis de la indeterminación del ordenamiento jurídico. Pero no es este el punto central del presente texto, ni tampoco he ofrecido mayores argumentos a favor de esta tesis escéptica.

Lo que, en cambio, he intentado argumentar, es que de esa indeterminación no se sigue que no existan otros criterios de corrección de la interpretación. Criterios que pueden ser llamados internos a un método interpretativo, ya que su funcionamiento depende de que el mismo intérprete se involucre en la práctica de usarlo. Cuando ello es el caso, esos métodos ofrecen, a través de sus directivas, criterios de corrección y razones para la acción. Ello significa que es posible decir que una interpretación es equivocada cuando el juez no ha seguido las directivas que definen el método interpretativo elegido. Además, la existencia de esos métodos ofrece un motivo para interpretar siguiendo la convención y, bajo ciertas condiciones, una justificación para hacerlo.

\section{REFERENCIAS BIBLIOGRÁFICAS}

Arena, F. J. (2011). Marmor on the Arbitrariness of Constitutive Conventions. Jurisprudence, I(2), 441-450.

Bayón Mohino, J. C. (1991). La normatividad del derecho. Deber jurídico y razones para la acción. Madrid: Centro de Estudios Constitucionales.

___ (2002). Derecho, convencionalismo y controversia. En Navarro, P. E. y Redondo, M. C. (Eds.), La relevancia del derecho (pp. 57-92). Barcelona: Gedisa.

Burge, T. (1975). On Knowledge and Convention. The Philosophical Review, 84(2), 249-255.

Celano, B. (2010). Fatti istituzionali, consuetudini, convenzioni. Roma: Aracne.

-_ (2010 [1995]). Consuetudini, convenzioni. En Celano, B. (Ed.), Fatti istituzionali, consuetudini, convenzioni (pp. 173-231). Roma: Aracne.

___ (2010 [1997]). Interazione strategica e convenzione. En Celano, B. (Ed.), Fatti istituzionali, consuetudini, convenzioni (pp. 281-322). Roma: Aracne.

-(2010[2003]).Laregoladiriconoscimento è una convenzione? En Celano, B. (Ed.), Fatti istituzionali, consuetudini, convenzioni (pp. 323-341). Roma: Aracne.

- (2017). Due problemi aperti della Teoria dell'interpretazione giuridica. Modena: Mucchi editore.

Chiassoni, P. (2004). Codici interpretativi: Progetto di voce per un Vademecum giuridico. Analisi e diritto, 2002-2003, 55-124. (2011). Técnicas de interpretación jurídica. Brevario para juristas. Madrid: Marcial Pons.

Coleman, J. L. (2001). The Conventionality Thesis. Philosophical Issues, 11(1), 354-387.

Dickson, J. (2007). Is the Rule of Recognition Really a Conventional Rule? Oxford Journal of Legal Studies, 27(3), 373-402.

Fuller, L. L. (1969 [1964]). The Morality of Law. New Haven: Yale University Press.

Gilbert, M. (2008). Social Convention Revisited. Topoi, 27(1/2), 5-16.

Guastini, R. (2012). El escepticismo ante las reglas replanteado. Discusiones, 11, 27-58.

___ (2014). Interpretar y argumentar (S. Álvarez Medina, Trans.). Madrid: Centro de Estudios Políticos y Constitucionales.

Kekes, J. (1989). Moral tradition and Individuality. Princeton: Princeton University Press.

Lewis, D. (1969). Convention. A Philosophical Study. Oxford: Basil Blackwell.

Marmor, A. (2001). Positive Law and Objective Values. Oxford-New York: Clarendon Press.

___ (2009). Social Conventions. From Language to Law. Princeton: Princeton University Press. 
Nino, C. S. (1992). Fundamentos de derecho constitucional. Buenos Aires: Astrea.

Postema, G. (1982). Coordination and Convention at the Foundation of Law. The Journal of Legal Studies, 11(1), 165-203.

(1987). The Normativity of Law. En Gavison, R. (Ed.), Issues in Contemporary Legal Philosophy. The Influence of H.L.A. Hart (pp. 81-104). Oxford: Clarendon Press.

Redondo, M. C. (2001). On Normativity in Legal Context. En Lagerspetz, E. (Ed.), On the Nature of Social and Institutional Reality. Jyväskylä: University of Jyväskylä Printing House.

Schiavello, A. (2010). Perché obbedire il diritto. La risposta convenzionalista e i suoi limiti. Pisa: ETS.
Searle, J. R. (1964). How to Derive «Ought» From «|s». The Philosophical Review, 73(1), 4358.

-_- (1969). Speech Acts. An Essay in the Philosophy of Language. London: Cambridge University Press. (1995). The Construction of Social Reality. New York: Free Press.

___ (2010). Making the Social World. The Structure of Human Civilization. OxfordNew York: Oxford University Press.

Shiner, R. A. (2010). Law and Its Normativity. En Patterson, D. (Ed.), A Companion to Philosophy of Law and Legal Theory (pp. 417-445). Oxford: Blackwell.

Vilajosana Rubio, J. M. (2010). El derecho en acción. La dimensión social de las normas jurídicas. Madrid: Marcial Pons. 